\title{
Interior and exterior sound field control using two dimensional higher-order variable-directivity sources
}

\author{
M. A. Polettia \\ Industrial Research Ltd, P.O. Box 31-310, Lower Hutt, Wellington 5040, New Zealand \\ T. D. Abhayapala and P. Samarasinghe \\ Research School of Engineering, ANU College of Engineering \& Computer Science, Building 115, Cnr North \\ and Daley Roads, Acton ACT 2600, The Australian National University, Canberra ACT 0200, Australia
}

(Received 11 August 2011; revised 17 February 2012; accepted 27 February 2012)

\begin{abstract}
Spatial sound reproduction systems aim to produce a desired sound field over a volume of space. At high frequencies, the number of loudspeakers required is prohibitive. This paper shows that the use of loudspeakers with up to $N$ th order directivity allows reproduction over $N$ times the bandwidth and produces a significantly attenuated exterior sound field. If the constraint on exterior cancellation of the field is removed, reproduction is possible over approximately $2 N$ times the bandwidth. The use of higher order loudspeakers thus allows a significant reduction in the number of loudspeaker units, at the expense of increased complexity in each unit. For completeness, results are included for the generation of an exterior field with or without cancellation of the interior field.
\end{abstract} (C) 2012 Acoustical Society of America. [http://dx.doi.org/10.1121/1.3699192]

PACS number(s): 43.38.Md, 43.60.Sx, 43.55.Jz, 43.60.Tj [NX]

Pages: $3814-3823$

\section{INTRODUCTION}

Sound reproduction systems aim to reproduce an arbitrary desired sound field within an array of loudspeakers. The desired sound field properties are either synthetically generated or recorded in the field using a microphone array. There are two main approaches to the derivation of the loudspeaker signals required to reproduce the sound field. The first is based on the polar two dimensional (2D) or spherical three dimensional (3D) harmonic expansions of a sound field. To record the sound field, the field is decomposed into a number of signals representing the polar or spherical harmonic responses, and these can be used to reconstruct the original sound field using a loudspeaker array. ${ }^{1-10}$ The second approach is based on the Kirchhoff-Helmholtz (KH) integral formula, which shows that a sound field may be produced within a volume of space by an infinite density of monopole and normally oriented dipole sources on the surface of that volume, and that the exterior field is zero. The sources both generate an interior field, and cancel any sound, leaving the desired reproduction volume. ${ }^{11-16}$ In practice, the $\mathrm{KH}$ approach is implemented using discrete arrays, and often employs only monopole loudspeakers, so that the exterior field is not canceled.

The accuracy of surround systems is governed by the wavelength and the size of the region over which accurate reproduction is required. For wave number $k$ and reproduction radius $r$ the number of required loudspeakers is given approximately by $L_{2} \approx 2 k r+1$ in the $2 \mathrm{D}$ case and by $L_{3} \approx(k r+1)^{2}$ in the 3D case. ${ }^{6,7}$ Hence, large numbers of loudspeakers are required for the reproduction of high frequencies over significant areas. For example, at $8 \mathrm{kHz}$ and for reproduction over a radius of $0.2 \mathrm{~m}, 60$ loudspeakers are

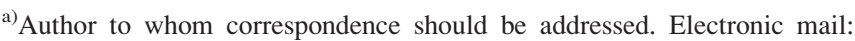
m.poletti@irl.cri.nz
}

required for the $2 \mathrm{D}$ case and 924 for the $3 \mathrm{D}$ case. It is typically infeasible to employ such numbers of loudspeakers in a sound reproduction setup in the home.

A second limitation of sound reproduction systems is that the loudspeaker sound fields reflect from room surfaces, creating a reverberant field inside the reproduction region, which corrupts the reproduced field. If variable directivity loudspeakers are used, ${ }^{11,17,18}$ as in the KH approach, the exterior field may be eliminated below the spatial Nyquist frequency, ${ }^{19}$ which in the $2 \mathrm{D}$ circular case ${ }^{20}$ is approximately ${ }^{18}$

$$
f_{1}=\frac{c(L-1)}{4 \pi r_{L}},
$$

where $c$ is the speed of sound, $L$ is the number of loudspeakers, and $r_{L}$ is the radius of the loudspeaker array. At this frequency, the loudspeakers are approximately half a wavelength apart. For practical numbers of loudspeakers, the spatial Nyquist frequency is typically low, and so exterior cancellation is only possible at bass frequencies.

If only fixed-directivity loudspeakers are available, the direct to reverberant ratio can be increased, reducing the effects of reverberation. ${ }^{21-24}$ Alternatively, the reverberation can be reduced by digital pre-compensation of the loudspeaker signals. ${ }^{25-29}$

The KH approach requires dipoles oriented normal to the reproduction surface. In Ref. 18 the use of general first order $2 \mathrm{D}$ sources, including dipoles tangential to the reproduction surface, was examined for improving the reproduction accuracy of circular arrays. The use of tangential dipoles was shown to improve sound field reproduction and to reduce the exterior field for frequencies close to and above the spatial Nyquist frequency Eq. (1).

This prompted an investigation of the use of higher order 2D sources, ${ }^{30}$ capable of producing polar responses $\cos (n \phi)$ and $\sin (n \phi)$ for $n \in[0,1, \ldots, N]$. These investigations showed 
that $N$ th order loudspeakers increase the bandwidth of accurate reproduction by a factor of $N$, or equivalently, increase the radius of accurate reproduction by $N$. This represents a considerable improvement over previous surround approaches, but at the expense of more complex loudspeaker units, which typically must be constructed using circular or spherical arrays of transducers. ${ }^{24,31-35}$

When a higher order sound reproduction system is used in a room, calibration may be used to reduce the reverberant field and produce more accurate reproduction. ${ }^{36}$ For example, a loudspeaker can reflect sound off a wall to produce directions of sound arrival other than loudspeaker angles. In this approach the room may be viewed as an additional resource for accurate sound reproduction, rather than a hindrance to it.

This paper considers the reproduction of sound fields using arrays of higher order loudspeakers. For simplicity we will consider the 2D case as in Ref. 30, and so the cylindrical expansions simplify to polar expansions. We also restrict our attention to free-field reproduction as we wish to examine the basic performance of higher order arrays in direct field sound reproduction.

We extend the work in Ref. 30 as follows: We first use more physically derived loudspeaker responses than were used in Ref. 30 and we also consider reproduction without the requirement for exterior cancellation, to determine if the resulting additional degrees of freedom produce an increase in the bandwidth of accurate reproduction. We also consider the complementary case of exterior sound field generation, with and without interior field cancellation, which is also described by the KH integral. In general, by linearity, an array may generate two separate interior and exterior sound fields.

We first provide a theoretical background for sound field reproduction in the $2 \mathrm{D}$ case, and introduce a more physically based model for a higher order (2D) source. We then derive the required loudspeaker weights using a least squares approach and verify the results by numerical simulations. We also derive the spatial Nyquist frequencies for the array for the cancellation and non-cancellation cases and for interior and exterior field generation, and quantify their accuracy by undertaking numerical simulations of sound field reproduction versus frequency.

\section{THEORETICAL BACKGROUND}

\section{A. Polar expansion of 2D sound fields}

The wave equation may be solved in polar coordinates $(r, \phi)$, where $r$ is the radius $r=\sqrt{x^{2}+y^{2}}$. The interior expansion of the sound field in a region $r<r_{0}$ that is homogeneous and free of sources is ${ }^{38}$

$$
p(r, \phi, k)=\sum_{m=-\infty}^{\infty} J_{m}(k r) A_{m}(k) e^{i m \phi}
$$

where the $A_{m}(k)$ are the interior sound field expansion coefficients, $J_{m}(\bullet)$ is the $m$ th order Bessel function, and $i=\sqrt{-1}$.

The corresponding expansion for regions $r>r_{0}$ exterior to any sound sources is

$$
p(r, \phi, k)=\sum_{m=-\infty}^{\infty} H_{m}(k r) B_{m}(k) e^{i m \phi},
$$

where the $B_{m}(k)$ are the exterior sound field expansion coefficients and $H_{m}(\bullet)=H_{m}^{(1)}(\bullet)$ is the $m$ th order Hankel function of the first kind, assuming a harmonic time dependence $\exp (-i \omega t)$. An important example of relevance later is the sound field due to a line source positioned at $\vec{r}_{s}=\left(r_{s}, \phi_{s}\right)$,

$$
p_{\text {line }}(r, \phi, k, t)=e^{-i \omega t} H_{0}^{(1)}\left(k\left\|\vec{r}-\vec{r}_{s}\right\|\right)=e^{-i \omega t} \begin{cases}\sum_{m=-\infty}^{\infty} J_{m}(k r) H_{m}^{(1)}\left(k r_{s}\right) e^{i m\left(\phi-\phi_{s}\right)}, & r<r_{s} \\ \sum_{m=-\infty}^{\infty} J_{m}\left(k r_{s}\right) H_{m}^{(1)}(k r) e^{i m\left(\phi-\phi_{s}\right)}, & r>r_{s} .\end{cases}
$$

\section{B. Description of higher order source}

A 2D higher order source positioned at the origin has the idealized form

$$
p_{n}(r, \phi, k)=H_{n}(k r) e^{i n \phi},
$$

where we use an index $n$ for the order of the source to distinguish it from the expansion coefficient $m$. From Eq. (3) this is a single term in the expansion of the exterior field. This form has been applied to the generation of sound fields in Ref. 30. However, it has a magnitude that tends to infinity as $k r$ tends to zero and the associated impulse response is not physically realizable. We therefore introduce a more physically derived source here.
A practical higher order loudspeaker would be one that is capable of radiating sound with far-field polar responses of the form $\cos (n \phi)$ and $\sin (n \phi)$. For the investigation in this paper we will consider sources that produce a complexvalued directivity of the form $\exp (\operatorname{in} \phi)$ to keep the mathematical description simple. The sin and cos responses are easily obtained from these complex-valued directivities. We consider a source at the origin, of radius $R$, with a radial velocity which can be written

$$
v_{r}(\phi)=V_{0} \sum_{m=-\infty}^{\infty} \alpha_{m} e^{i m \phi},
$$

where the $\alpha_{m}$ are the Fourier series coefficients. The 2D exterior field has the form of Eq. (3), from which the associated radial velocity is 


$$
v_{r}(r, \phi, k)=\frac{-i}{\rho c} \sum_{m=-\infty}^{\infty} B_{m} H_{m}^{\prime}(k r) e^{i m \phi} .
$$

This must be equal to the radial velocity in Eq. (6) for $r=R$. Hence the sound pressure is ${ }^{37}$

$$
p(r, \phi, k, t)=i \rho c V_{0} \sum_{m=-\infty}^{\infty} \alpha_{m} \frac{H_{m}(k r)}{H_{m}^{\prime}(k R)} e^{i m \phi} e^{-i \omega t}
$$

We see that each mode has a phase variation $\exp (\operatorname{im} \phi)$ and a radial variation $H_{m}(k r)$, with a scale factor $\alpha_{m} / H_{m}^{\prime}(k R)$. This factor ensures that the $m$ th order source has a magnitude of zero at $0 \mathrm{~Hz}$, and the associated impulse response is that of a high pass function with finite energy.

In order to produce a single higher order source response $\exp (i n \phi)$ the surface velocity must satisfy

$$
\alpha_{m}= \begin{cases}1, & m=n \\ 0, & m \neq n\end{cases}
$$

The mode responses $\rho c H_{n}(k r) / H_{n}^{\prime}(k R)$ are shown for $n=0$ to $n=5$ in Fig. 1. The source produces a roll-off for frequencies below, approximately, $k R=n$, or

$$
f_{m}=\frac{n c}{2 \pi R} .
$$

For example, this produces $f_{n}=433 \mathrm{~Hz}$ for $n=2$ and $f_{n}=1083 \mathrm{~Hz}$ for $n=5$. We note that, since sound reproduction systems work well at low frequencies with omnidirectional loudspeakers, higher order modes are only required at high frequencies, and hence the low magnitude of the high order responses at low frequencies is not a significant limitation. Note also that a practical higher order source using discrete drivers would only produce the desired response up to a finite frequency, above which aliasing would occur.

\section{Description of translated higher order sources}

The sound field produced by an ideal higher order source [Eq. (5)] positioned at $\left(r_{s}, \phi_{s}\right)$ can be expressed relative to the origin using the cylindrical addition theorem, ${ }^{38}$

$$
p_{n}\left(r, \phi, r_{s}, \phi_{s}, k\right)=H_{n}\left(k r_{0}\right) e^{i n \beta_{s}}= \begin{cases}\sum_{m=-\infty}^{\infty} J_{m}(k r) H_{m+n}\left(k r_{s}\right) e^{i m\left(\phi-\phi_{s}\right)}, & r<r_{s} \\ \sum_{m=-\infty}^{\infty} J_{m+n}\left(k r_{s}\right) H_{m}(k r) e^{i m\left(\phi-\phi_{s}\right)}, & r>r_{s}\end{cases}
$$

where see (Fig. 2) $r_{0}=\left\|\vec{r}-\vec{r}_{s}\right\|$ and $\beta_{s}$ is the angle measured between $\vec{r}_{0}$ and the source vector $\vec{r}_{s}$,

$$
\beta_{s}=\arctan \left[\frac{y_{\mathrm{rot}}}{r_{s}-x_{\mathrm{rot}}}\right],
$$

where

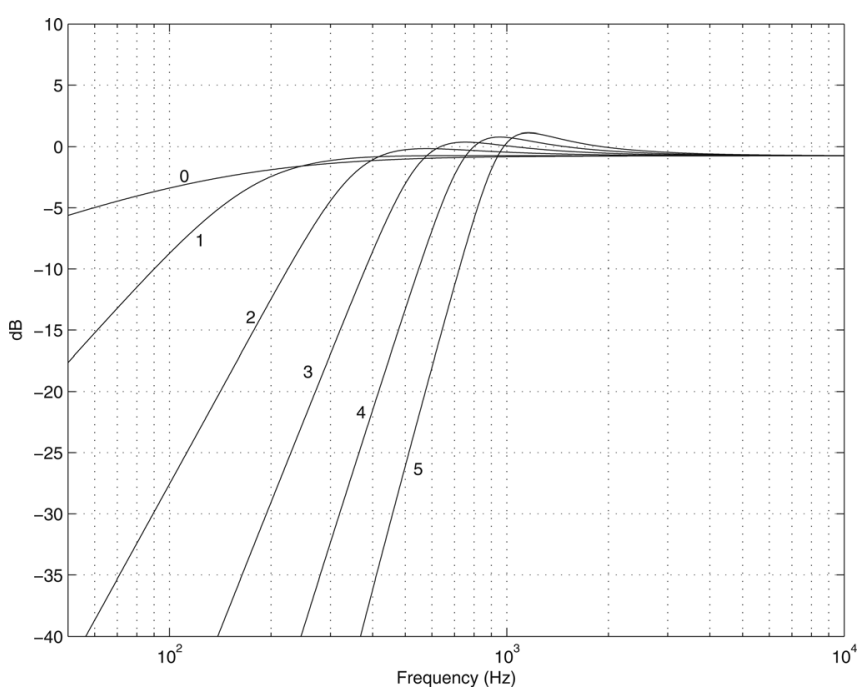

FIG. 1. Cylinder mode responses for $R=0.25, \Delta=\pi / 50$.

$$
x_{\mathrm{rot}}=x \cos \phi_{0}+y \sin \phi_{0}
$$

and

$$
y_{\text {rot }}=-x \sin \phi_{0}+y \cos \phi_{0} .
$$

Equation (11) can be normalized by $H_{n}^{\prime}(k R)$ to produce the more physically reasonable form [Eq. (8)].

In practice, the infinite sums in Eq. (11) can be replaced with finite summations, $m \in[-M, M]$, due to the high pass

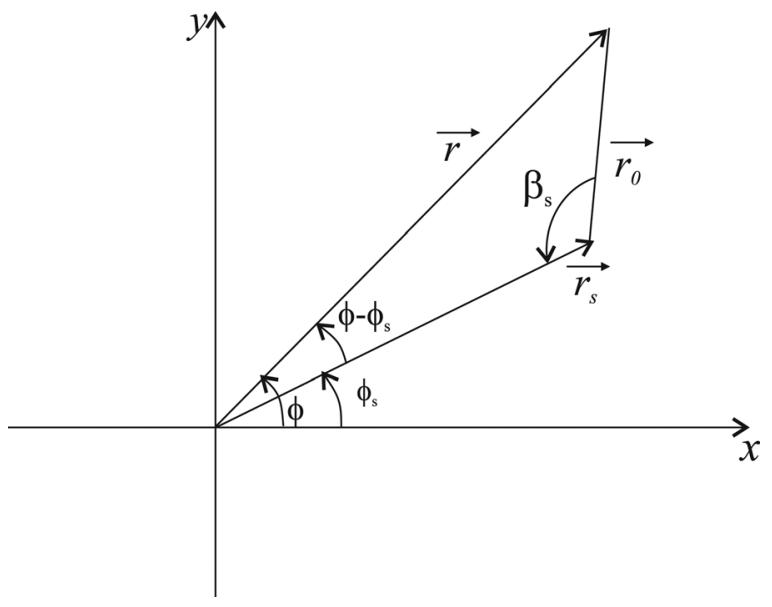

FIG. 2. Angles for application of the addition theorem. 
nature of the Bessel functions. For the interior expansion, $J_{m}(k r)$ is small for $m>k r$ and hence $M=\lceil k r\rceil$, where $\lceil$. denotes rounding up to the next highest integer, is sufficient. $^{6,39}$ A more accurate derivation for the minimum required order is $m>e k r / 2$, where $e=2.718$ is Euler's number, and we will use this form in deriving the array spatial Nyquist frequencies in the next section. ${ }^{40}$ For the exterior expansion $J_{m+n}\left(k r_{s}\right)$ is small for $m-n>k r_{s}$ and hence $M=\mathrm{N}+\left\lceil k r_{s}\right\rceil$ (more accurately $M=\mathrm{N}+e k r_{s} / 2$ ).

In both interior and exterior cases, mode orders higher than the above-mentioned approximations are required to describe the sound field near the source radius $r=r_{s}$, and the required order is larger at higher orders $n$. This can be shown from the angle-averaged normalized truncation error of Eq. (11), defined as $6,17,21$

$$
\bar{\varepsilon}_{M}(n, k r)=\frac{\int_{0}^{2 \pi}\left|p_{n}\left(r, \phi, r_{s}, \phi_{s}, k\right)-p_{n M}\left(r, \phi, r_{s}, \phi_{s}, k\right)\right|^{2} d \phi}{\int_{0}^{2 \pi}\left|p_{n}\left(r, \phi, r_{s}, \phi_{s}, k\right)\right|^{2} d \phi},
$$

where $p_{n M}\left(r, \phi, r_{s}, \phi_{s}, k\right)$ is the $M$ th order truncated form of Eq. (11). The truncation error can be shown to be

$$
\bar{\varepsilon}_{M}(n, k r)=\left\{\begin{array}{cl}
1-\frac{\sum_{m=-M}^{M} J_{m}^{2}(k r)\left|H_{m+n}\left(k r_{s}\right)\right|^{2}}{\sum_{m=-\infty}^{\infty} J_{m}^{2}(k r)\left|H_{m+n}\left(k r_{s}\right)\right|^{2}}, & r<r_{s} \\
1-\frac{\sum_{m=-M}^{M} J_{m+n}^{2}\left(k r_{s}\right)\left|H_{m}(k r)\right|^{2}}{\sum_{m=-\infty}^{\infty} J_{m+n}^{2}\left(k r_{s}\right)\left|H_{m}(k r)\right|^{2}}, & r>r_{s} .
\end{array}\right.
$$

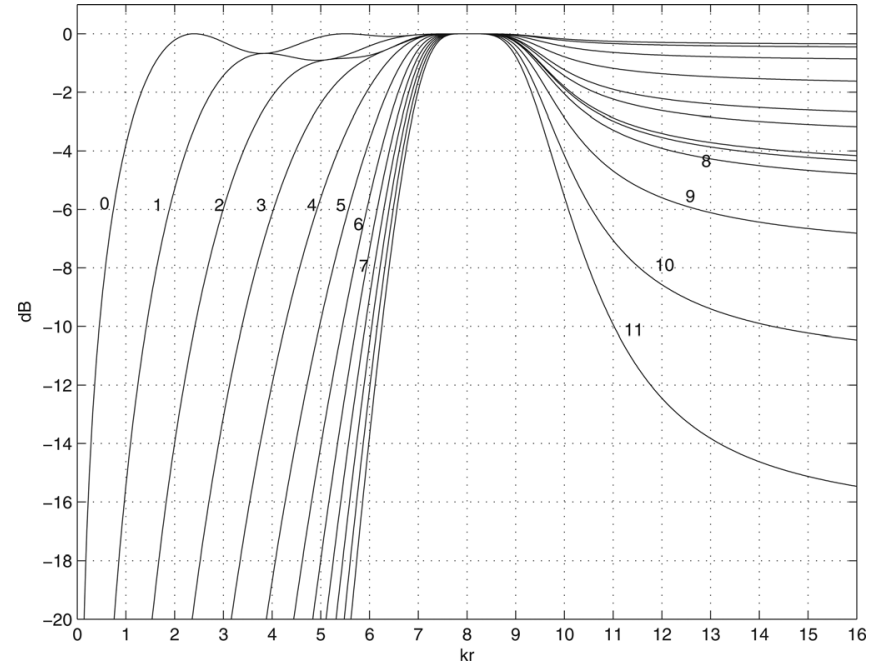

FIG. 3. Truncation error for $N=3$ source $k r_{s}=8$.

This is shown for $N=3$ in Fig. (3) for a source with $k r_{s}=8$, which may be compared with the $N=0$ case in Ref. 17 . The $m>k r$ approximation is valid for $k r \ll k r_{s}$, but for radii close to the source, the required order tends to infinity. Similarly, the exterior truncation error is small for $m>N+k r_{s}=11$ for radii that are not close to $r_{s}$.

A general higher order source is a single loudspeaker unit that can produce all orders of response up to a given value $N$. A single $N$ th order source with coefficients $w_{n}, n \in[-\mathrm{N}, \mathrm{N}]$ produces the sound field

$$
p_{N}\left(r, \phi, r_{s}, \phi_{s}\right)=\sum_{n=-N}^{N} w_{n} \frac{H_{n}\left(k\left\|\vec{r}-\vec{r}_{s}\right\|\right)}{H_{n}^{\prime}(k R)} e^{i n \beta_{s}}
$$

From Eq. (11) the single higher order source has an expansion relative to the origin given by

$$
p_{N}\left(r, \phi, r_{s}, \phi_{s}, k\right)= \begin{cases}\sum_{m=-\infty}^{\infty} J_{m}(k r) e^{i m\left(\phi-\phi_{s}\right)}\left[\sum_{n=-N}^{N} w_{n} \frac{H_{m+n}\left(k r_{s}\right)}{H_{n}^{\prime}(k R)}\right], & r<r_{s} \\ \sum_{m=-\infty}^{\infty} H_{m}(k r) e^{i m\left(\phi-\phi_{s}\right)}\left[\sum_{n=-N}^{N} w_{n} \frac{J_{m+n}\left(k r_{s}\right)}{H_{n}^{\prime}(k R)}\right], & r>r_{s} .\end{cases}
$$

The superposition of $L$ higher order sources that are controlled by individual weights $w_{n, l}, l \in[1, L]$ and which are arranged on a circle of radius $r_{s}=r_{L}$, and at angles $\phi_{l}$ produces the sound field

$$
\hat{p}(r, \phi, k)= \begin{cases}\sum_{m=-\infty}^{\infty} J_{m}(k r) e^{i m \phi}\left[\sum_{l=1}^{L} \sum_{n=-N}^{N} w_{n, l} \frac{H_{m+n}\left(k r_{L}\right)}{H^{\prime}{ }_{n}(k R)} e^{-i m \phi_{l}}\right], & r<r_{L} \\ \sum_{m=-\infty}^{\infty} H_{m}(k r) e^{i m \phi}\left[\sum_{l=1}^{L} \sum_{n=-N}^{N} w_{n, l} \frac{J_{m+n}\left(k r_{L}\right)}{H^{\prime}{ }_{n}(k R)} e^{-i m \phi_{l}}\right], & r>r_{L},\end{cases}
$$


which is applied to approximate a desired interior or exterior sound field [Eq. (2) or Eq. (3)].

\section{SOUND REPRODUCTION}

In order to reproduce a desired sound field with an array of $L$ sources, each producing up to $N$ th order polar responses, the weights $w_{n, l}$ of each radiating mode must be determined. There are two approaches to this problem. In the first, a continuous distribution of higher order sources is assumed and the higher order source weights may be determined in a manner similar to Ref. 18. Such a solution is the generalization of the simple source solution. In the second approach, the weights $w_{n, l}$ for the discrete array are determined directly using a least squares error minimization. We follow this approach here. We first consider the interior and exterior cases without specifying control of the sound field in the other region, and then consider the general case of producing separate interior and exterior sound fields, one of which may be zero.

\section{A. Interior case}

For the creation of an interior field with no exterior control we require the weighted sum of the higher order source fields to produce a desired interior field with arbitrary interior expansion coefficient $A_{m}$. The higher order sources produce polar responses up to order $N$, and the order of the expansion describing the desired sound field is $M$. Hence, from Eqs. (2) and (19), this yields, for each mode,

$$
\sum_{n=-N}^{N} \frac{H_{n+m}\left(k r_{L}\right)}{H_{n}^{\prime}(k R)} \sum_{l=1}^{L} w_{n, l} e^{-i m \phi_{l}}=A_{m}, \quad m \in[-M, M] .
$$

This set of equations may be put in matrix form

$$
\mathbf{H} w=a,
$$

where $\mathbf{H}$ is a $2 M+1$ by $(2 N+1) L$ matrix, $w$ is a $(2 N+1) L$ by one vector of weights, and $a$ is a $2 M+1$ vector of interior coefficients. This may be solved for the interior field solution. The solution

$$
w=\mathbf{H}^{H}\left[\mathbf{H} \mathbf{H}^{H}+\lambda \mathbf{I}\right]^{-1} a,
$$

where $\mathbf{I}$ is the $2 M+1$ by $2 M+1$ identity matrix, exists for $2 M+1 \leq \mathrm{L}(2 N+1)$. For $\lambda=0$ this is the minimum energy solution $^{6}$ and $\lambda$ can be used to reduce the weight solutions for cases where $\mathbf{H}$ has small singular values. The maximum range of modes is controlled when $2 M+1=L(2 N+1)$. However, the $H_{n+m}\left(k r_{L}\right) / H_{n}^{\prime}(k R)$ terms, and the condition number of $\mathbf{H}$, can become large for large $m$ at low frequencies, and this can produce large amplitude weight solutions. Furthermore, the conditioning of $\mathbf{H}$ becomes poor when it is square. ${ }^{17,21,41} \mathrm{In}$ addition to the regularization of the solution, we use the minimum required mode order for reproducing the interior sound field, and limit the maximum mode order to $2 M+1<L(2 N+1)$. At low frequencies the required order $M$ to reproduce the sound field within the array is ${ }^{40}$

$$
M(k)=\left\lceil\frac{e k r_{L}}{2}\right\rceil .
$$

The order rises with frequency, and is limited to a maximum value,

$$
2 M_{\max }+1=\beta L(2 N+1), \quad \beta<1 .
$$

The approximate spatial Nyquist frequency of the array occurs when the required order $M(k)$ reaches the maximum $M_{\max }$,

$$
f_{N I}=\frac{c(\beta L(N+1 / 2)-1 / 2)}{e \pi r_{L}} .
$$

This value is more conservative than that derived using the $m=k r$ approximation and specifies when the reproduction error begins to rise. However, the array can still produce relatively accurate reproduction for frequencies above, but close to, Eq. (24). Using a value of $\beta$ below one reduces the spatial Nyquist frequency slightly, but the interior reproduction error and exterior level above the Nyquist frequency are lower and hence the performance of the system more robust. Equation (25) is similar to Eq. (1) for $N=0$ and $\beta=1$ (the difference being due to the use of the $e k r_{L} / 2$ rather than the $k r_{L}$ approximation).

Above the spatial Nyquist frequency, the maximum radius over which accurate reproduction is possible is smaller than the array radius $r_{L}$, and is given by

$$
r_{\max }(f)=\frac{c(\beta L(N+1 / 2)-1 / 2)}{e \pi f} .
$$

\section{B. Exterior case}

For the creation of an exterior field, with no constraint on the interior field, from Eq. (19), we require

$$
\sum_{n=-N}^{N} \frac{J_{m+n}\left(k r_{L}\right)}{H_{n}^{\prime}(k R)} \sum_{l=1}^{L} w_{n, l} e^{-i m \phi_{l}}=B_{m}, \quad m \in[-M, M]
$$

which can be written

$$
\mathbf{J} w=b,
$$

where $\mathbf{J}$ is a $2 M+1$ by $(2 N+1) L$ matrix, $w$ is a $(2 N+1) L$ by one vector of weights for exterior reproduction, and $b$ is a $2 M+1$ vector of interior coefficients. The solution is obtained using Eq. (22).

As mentioned earlier, the required mode order $M$ is determined at low frequencies from the exterior field constraint,

$$
M_{E}(k)=\left\lceil N+\frac{e k r_{L}}{2}\right\rceil .
$$

The maximum value is limited as in Eq. (24), yielding the spatial Nyquist frequency, 


$$
f_{N E}=\frac{c(\beta L-1)(N+1 / 2)}{e \pi r_{L}} .
$$

Note that $f_{N E}$ is slightly lower than $f_{N I}$, due to the additional term $N$ in Eq. (29).

\section{Interior/exterior case}

In the general case, we require the weighted sum of the higher order source fields to produce a desired interior field with arbitrary interior expansion coefficient $A_{m}$ and a desired exterior field with arbitrary interior expansion coefficient $B_{m}$. One of these sets of coefficients may be zero.

Equations (19) and (27) may be combined as

$$
\left[\begin{array}{l}
\mathbf{H} \\
\mathbf{J}
\end{array}\right] w=\boldsymbol{\Psi}_{w}=\left[\begin{array}{l}
a \\
b
\end{array}\right]
$$

which can be solved in a similar manner to Eq. (22) using $\boldsymbol{\Psi}$ instead of $\mathbf{H}$. The matrix $\boldsymbol{\Psi}$ is $4 M+2$ by $(2 N+1) L$. The required interior order is given in Eq. (23) and the exterior order in Eq. (29). At low frequencies these values may be used, and the maximum total mode order limited to $2\left(M_{I}+M_{E}\right)+1=\beta L(2 N+1)$. The spatial Nyquist frequency is then

$$
f_{N I E}=\frac{c(\beta L-1)\left(N+\frac{1}{2}\right)}{2 \pi e r_{L}} .
$$

This differs from the result in Ref. 30, which did not include the term $N$ in Eq. (29), did not include $\beta$, and which ignored the order $n=0$ source terms. However, the results in Sec. IV $\mathrm{C}$ will show that Eq. (32) is a good indicator of the frequency above which point the reproduction error starts to increase.

The maximum region of reproduction for interior/exterior control for frequencies above the spatial Nyquist frequency is

$$
r_{M I E}(f)=\frac{c(\beta L-1)\left(N+\frac{1}{2}\right)}{2 \pi e f} .
$$

From Eqs. (32) and (33), we see that the $N$ th order spatial Nyquist frequency and the radius of accurate reproduction are approximately $N$ times those of a zeroth or first order array $^{30}$ [Eq. (1)].

\section{RESULTS}

\section{A. Reproduction error in the reproduction region}

The accuracy of interior sound field reproduction with radius may be quantified by the angle-averaged relative error, $6,17,18,21$

$$
\bar{\varepsilon}(k r)=\frac{\int_{0}^{2 \pi}|p(r, \phi, k)-\hat{p}(r, \phi, k)|^{2} d \phi}{\int_{0}^{2 \pi}|p(r, \phi, k)|^{2} d \phi} .
$$

Substituting from Eqs. (2) and (19) and employing the orthonormality of the phase modes yields

$$
\bar{\varepsilon}(k r)=\frac{\sum_{m=-\infty}^{\infty} J_{m}^{2}(k r)\left|A_{m}(k)-Z_{\mathrm{I}}(m)\right|^{2}}{\sum_{m=-\infty}^{\infty} J_{m}^{2}(k r)\left|A_{m}(k)\right|^{2}},
$$

where

$$
Z_{I}(m)=\sum_{l=1}^{L}\left[\sum_{n=-N}^{N} w_{n, l} H_{m+n}\left(k r_{L}\right)\right] e^{-i m \phi_{l}} .
$$

Similarly, the exterior angle-averaged reproduction error is

$$
\bar{\varepsilon}(k r)=\frac{\sum_{m=-\infty}^{\infty} J_{m}^{2}(k r)\left|B_{m}(k)-Z_{E}(m)\right|^{2}}{\sum_{m=-\infty}^{\infty} J_{m}^{2}(k r)\left|B_{m}(k)\right|^{2}},
$$

where

$$
Z_{E}(m)=\sum_{l=1}^{L}\left[\sum_{n=-N}^{N} w_{n, l} J_{m+n}\left(k r_{L}\right)\right] e^{-i m \phi_{l}} .
$$

The radial error can be summed over a range of radii to produce an average over volume (for height invariance), which will be used to quantify the average performance of higher order arrays as a function of frequency in Sec. IV C.

\section{B. Sound level in cancellation region}

The cancellation of the sound field in the nonreproduction zone may be quantified by the average over angle of the magnitude squared of the approximate sound pressure $\hat{p}(r, \phi, k)$, relative to the average magnitude squared pressure due to the desired sound field $p(r, \phi, k)$ at the higher order source array radius $r_{L}{ }^{17,18,21}$ For interior reproduction, the exterior relative level is

$$
\bar{\Gamma}_{E}(k r)=\frac{\int_{0}^{2 \pi}|\hat{p}(r, \phi, k)|^{2} d \phi}{\int_{0}^{2 \pi}\left|p\left(r_{L}, \phi, k\right)\right|^{2} d \phi}, \quad r>r_{L} .
$$

Substituting from Eq. (2) and Eq. (19) yields

$$
\bar{\Gamma}_{E}(k r)=\frac{\sum_{m=-\infty}^{\infty}\left|H_{m}(k r)\right|^{2}\left|Z_{E}(m)\right|^{2}}{\sum_{m=-\infty}^{\infty}\left|A_{m}(k)\right|^{2} J_{m}^{2}\left(k r_{L}\right)}, \quad r>r_{L}
$$

Similarly, for exterior reproduction, the relative interior level is 


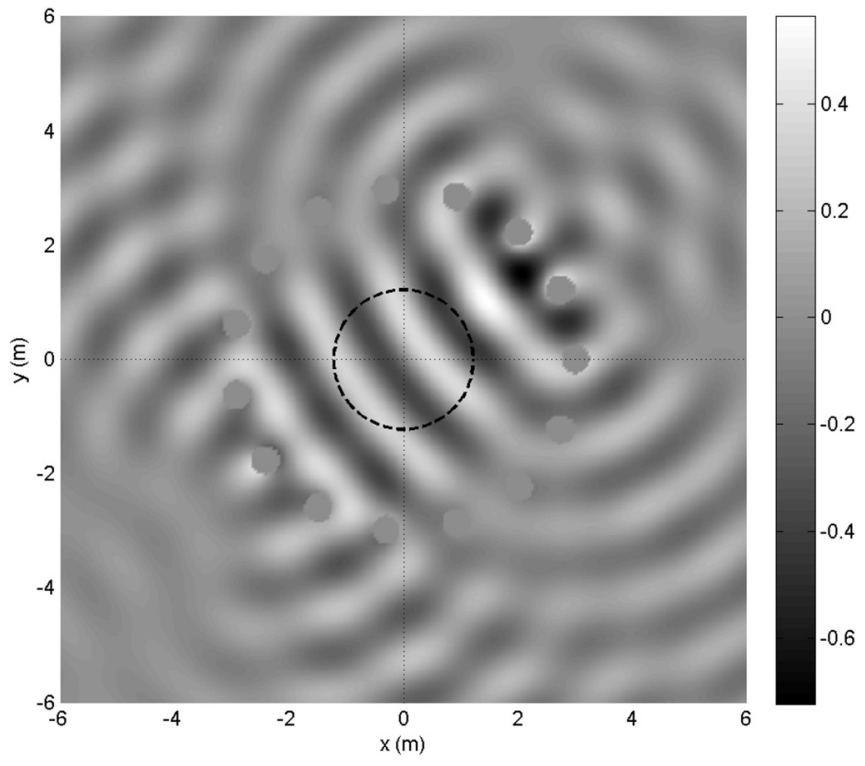

FIG. 4. $N=1, L=15, f_{0}=250 \mathrm{~Hz}$, with exterior cancellation.

$$
\bar{\Gamma}_{I}(k r)=\frac{\sum_{m=-\infty}^{\infty}\left|J_{m}(k r)\right|^{2}\left|Z_{I}(m)\right|^{2}}{\sum_{m=-\infty}^{\infty}\left|B_{m}(k)\right|^{2}\left|H_{m}\left(k r_{L}\right)\right|^{2}}, \quad r<r_{L} .
$$

The sound level can also be averaged over radius to produce a single figure of merit at a given frequency

\section{Simulations}

We will assume an array of $L=15$ higher order sources, each of radius $R=0.25 \mathrm{~m}$, in a circular array with a radius of $r_{L}=3 \mathrm{~m}$. We consider mode orders up to $N=4$ and a mode order regulation factor of $\beta=0.75$, which produces a slight reduction in spatial Nyquist frequency and accurate reproduction radius, but lower reproduction errors above the spa-

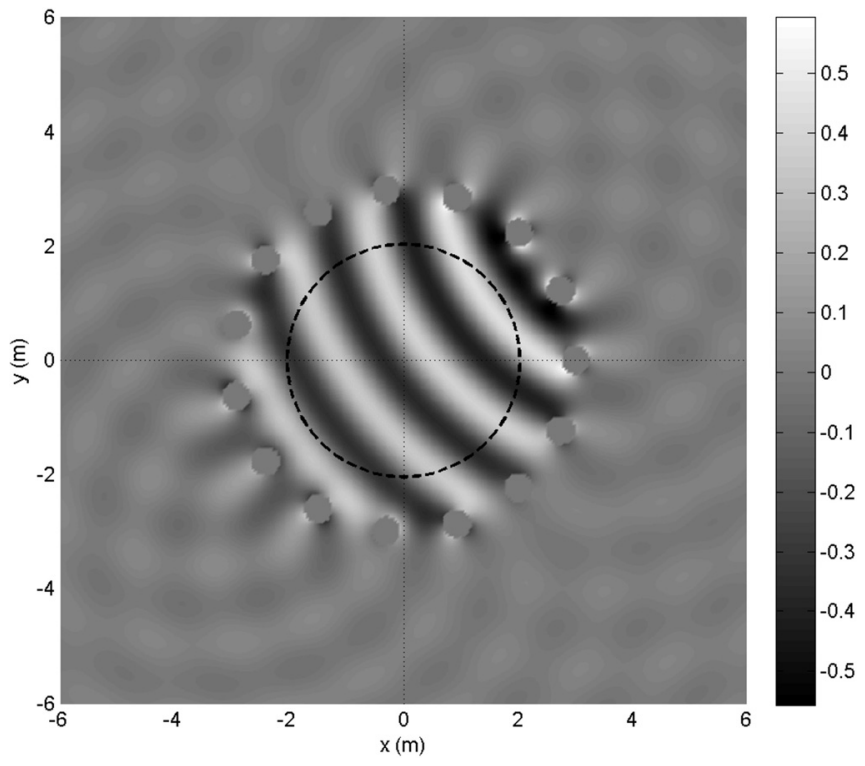

FIG. 5. $N=2, f_{0}=250 \mathrm{~Hz}$, with exterior cancellation.

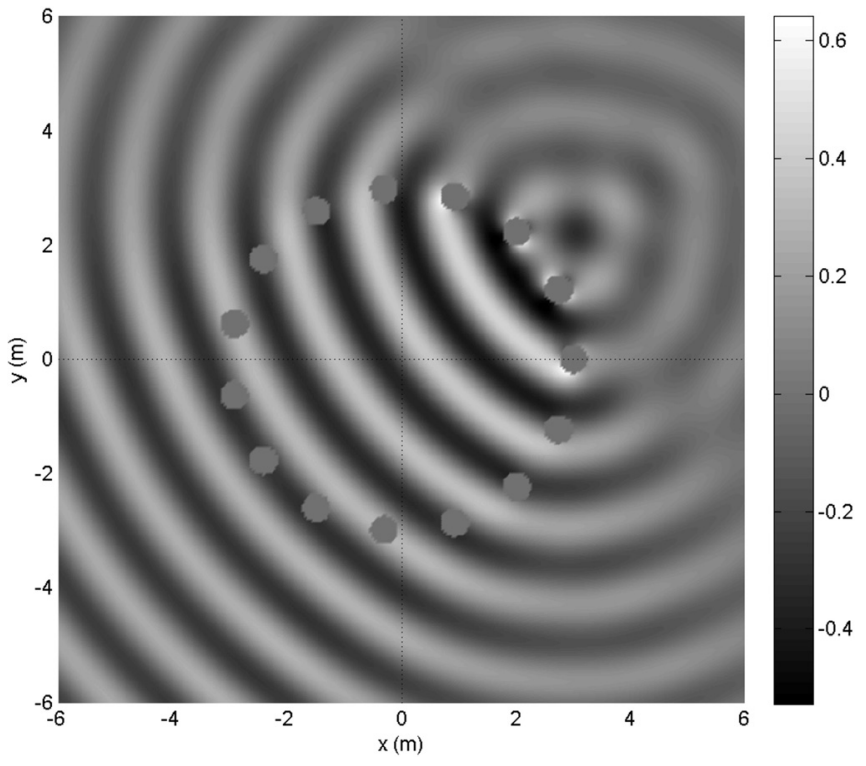

FIG. 6. $N=2, f_{0}=250 \mathrm{~Hz}$, without exterior cancellation.

tial Nyquist frequency. We use a regularization parameter $\lambda=0.001$ for obtaining the solutions for the weights according to Eq. (22). The desired source is a line source [Eq. (4)], positioned at a radius of $6 \mathrm{~m}$ and an angle of $36^{\circ}$, halfway between the higher order sources at $24^{\circ}$ and $48^{\circ}$.

Figure 4 shows the sound field produced by a first order array with attempted exterior cancellation at a frequency of $250 \mathrm{~Hz}$, well above the array spatial Nyquist frequency (100 $\mathrm{Hz}$ ). The radius of reproduction is $r_{M I E}=1.0 \mathrm{~m}$, shown as the dashed circle. The sound field is accurate within this radius, but is less accurate outside it, and the exterior sound field is non-zero. Figure 5 shows the sound field for the second order source array at the same source frequency, for which the spatial Nyquist frequency is $170 \mathrm{~Hz}$. The reproduction radius is $r_{M I E}=2.0 \mathrm{~m}$, and reproduction as accurate over twice the reproduction region in Fig. 4. The value of $r_{M I E}$ is somewhat conservative, as the field appears approximately correct out

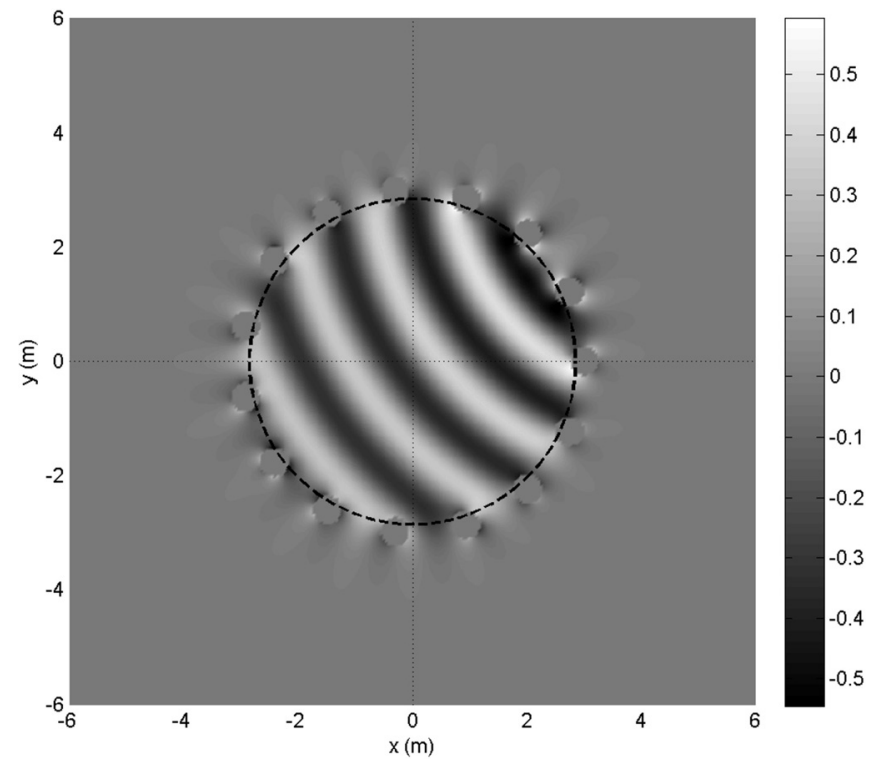

FIG. 7. $N=3, f_{0}=250 \mathrm{~Hz}$, with exterior cancellation. 


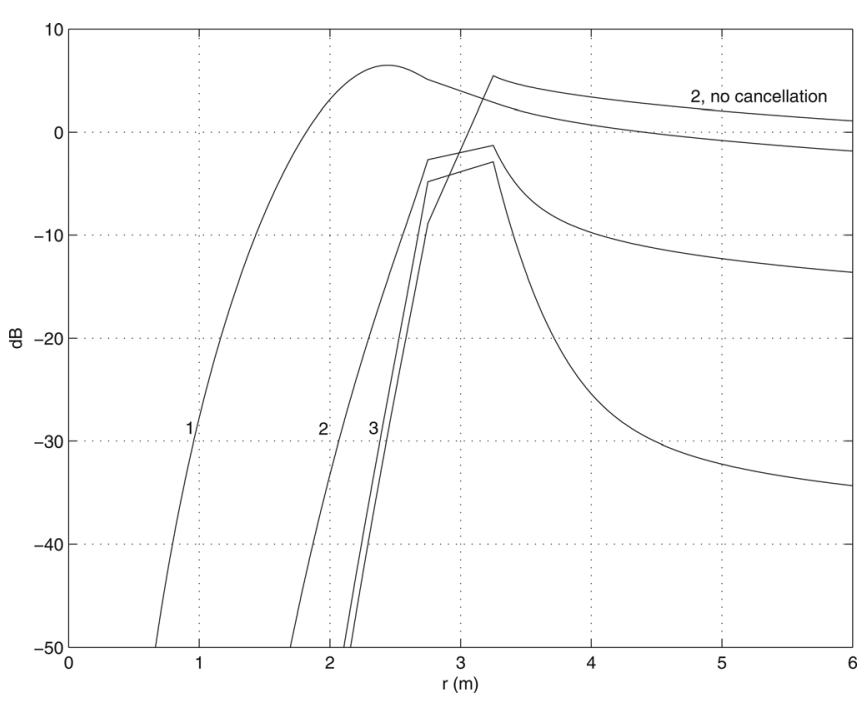

FIG. 8. Interior radial reproduction error and exterior radial level for $N=1-3, f_{0}=250 \mathrm{~Hz}, L=15$ with exterior cancellation. The $N=2$ without exterior cancellation is also shown.

to the array radius of $3 \mathrm{~m}$. However, the exterior field is nonzero, and the array is not able to completely meet the exterior cancellation constraint below the spatial Nyquist frequency, although the exterior amplitude is lower than in Fig. 4.

Figure 6 shows the sound field for $N=2$ but without exterior cancellation. The spatial Nyquist frequency is 370 $\mathrm{Hz}$, which is larger than the $250 \mathrm{~Hz}$ source frequency. Reproduction is now accurate throughout the interior of the array, but the exterior field is larger than it is in Fig. 5, particularly where the sound field radiates out of the array at angles near $216^{\circ}$.

Figure 7 shows the field with exterior cancellation, for $N=3$, producing a spatial Nyquist frequency of $310 \mathrm{~Hz}$. The field is now accurate within the array and the exterior field is small. The exterior level is below $-30 \mathrm{~dB}$ for radii greater than $4.5 \mathrm{~m}$ (1.5 $\mathrm{m}$ from the sources). This is shown in Fig. 8, which displays the angle-averaged radial errors for all of the above-mentioned cases. The second order array produces accurate reconstruction over two times the radius of the first

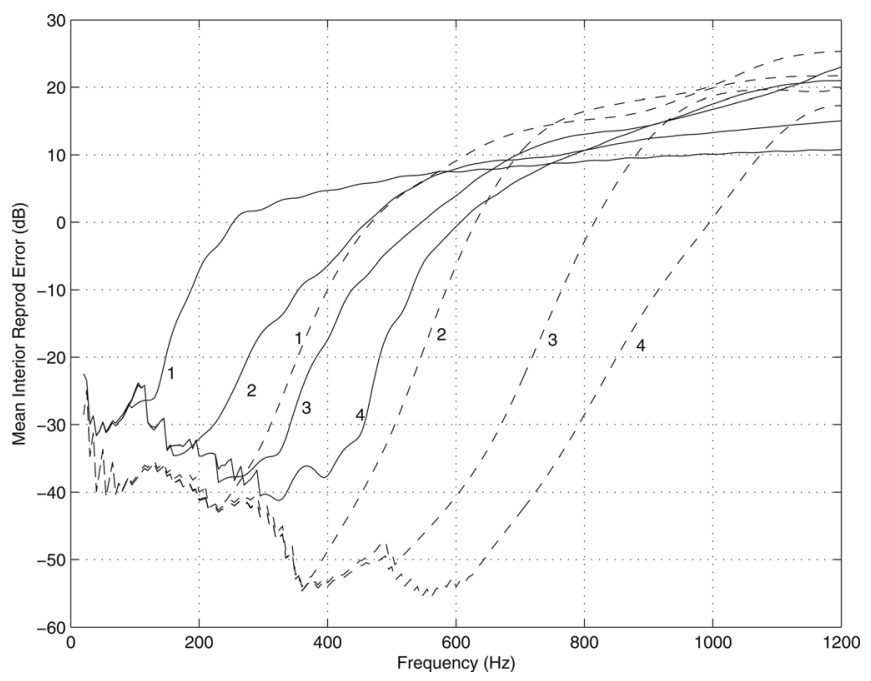

FIG. 9. Interior reproduction: Interior error with exterior cancellation (-) and without (-- -).
TABLE I. Spatial Nyquist frequencies, $\beta=0.75$, including the results from Ref. 30.

\begin{tabular}{ccccc}
\hline \hline$N$ & $f_{N I}(\mathrm{~Hz})$ & $f_{N E}(\mathrm{~Hz})$ & $f_{\text {NIE }}(\mathrm{Hz})$ & $f_{\text {NIE }}(\mathrm{Hz})(\mathrm{Ref} .30)$ \\
\hline 1 & 220 & 210 & 100 & 130 \\
2 & 370 & 340 & 170 & 260 \\
3 & 520 & 480 & 240 & 400 \\
4 & 670 & 620 & 310 & 540 \\
\hline \hline
\end{tabular}

order array, but the third order array produces only around 2.5 times the reproduction radius. This is because the maximum radius of accurate reproduction is close to the higher order source radius, and the approximate mode limitation [Eqs. (23) and (29)] are less applicable to the higher order source expansions. For the $N=2$ case without exterior cancellation the interior error is smaller than the third order array with cancellation, but the exterior level is higher than all other configurations.

Figure 9 shows the interior reproduction error as a function of frequency for both exterior cancellation and no cancellation. This was calculated as the average of the radial error from 0 to $2.5 \mathrm{~m}, 0.5 \mathrm{~m}$ away from the higher order sources. The reproduction error begins to rise above the spatial Nyquist frequencies given in Table I. In particular, the requirement for exterior cancellation produces approximately half the spatial Nyquist frequency of the no-cancellation case. The mean exterior levels are shown in Fig. 10. The levels with cancellation are small below the spatial Nyquist frequency, but the weight magnitudes tend to increase above it, producing mean exterior levels over $10 \mathrm{~dB}$. Without exterior cancellation, the weight magnitudes and exterior levels are up to $20 \mathrm{~dB}$ higher than the exterior cancellation levels. The weight magnitudes and the exterior sound level can be reduced by increasing the regularization parameter $\lambda$. Alternatively, a simpler sound reproduction strategy could be used for frequencies above the spatial Nyquist frequency, such as a fixed directivity approach, which provides some reduction of the reverberant field. ${ }^{21}$ However, a low-order fixed

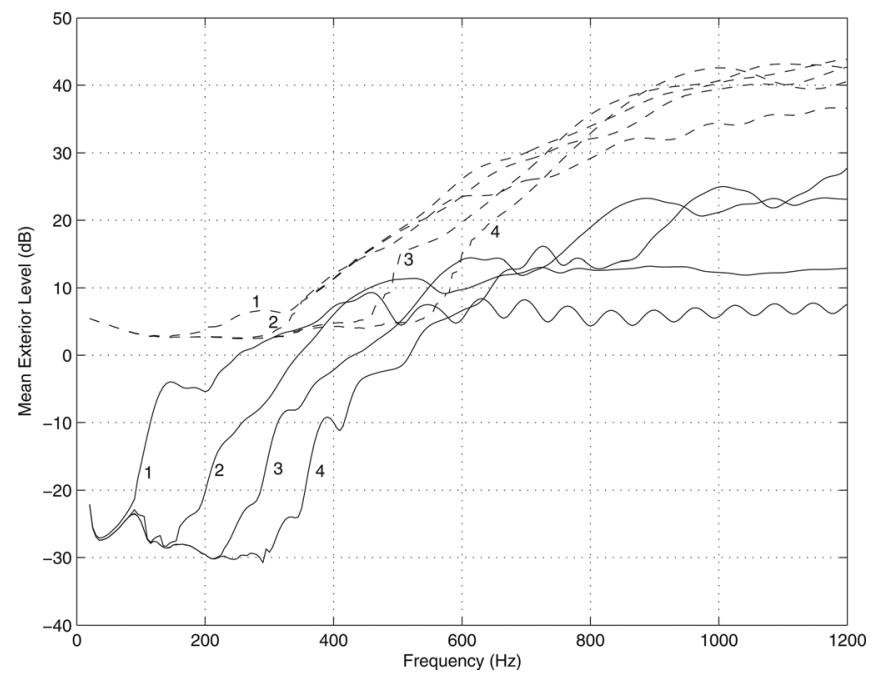

FIG. 10. Interior reproduction: Exterior mean level with exterior cancellation (-) and without (-- -). 


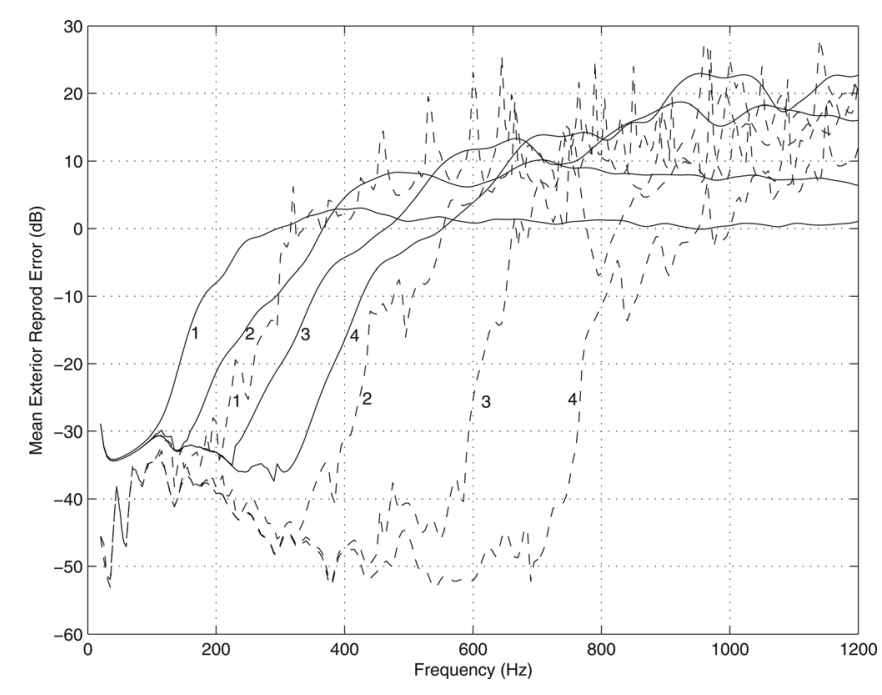

FIG. 11. Exterior reproduction: Exterior mean error with interior cancellation (-) and without (-- -).

directivity solution would produce a smaller zone of accurate reproduction than the regularized higher order solution.

Figure 11 shows the reproduction error for the production of an exterior sound field, using a source radius of $1.5 \mathrm{~m}$, within the source array. The spatial Nyquist frequencies with cancellation are essentially the same as for the cancellation case in Fig. 9. They are relatively conservative values which define approximately the frequency where the reproduction error begins to rise, even though the accuracy remains reasonable (e.g., below -20 dB) for a range of frequencies above the spatial Nyquist frequency. Note that in the exterior reproduction case without interior field cancellation, there are peaks in the error without interior cancellation. These peaks vary with the source position. The interior level without interior cancellation is larger than that with cancellation, as shown in Fig. 12, and produces peaks at the same frequencies as those where the exterior error is large. The precise cause of these peaks is currently unclear: they do not appear to be related in a simple manner to the internal resonant modes of

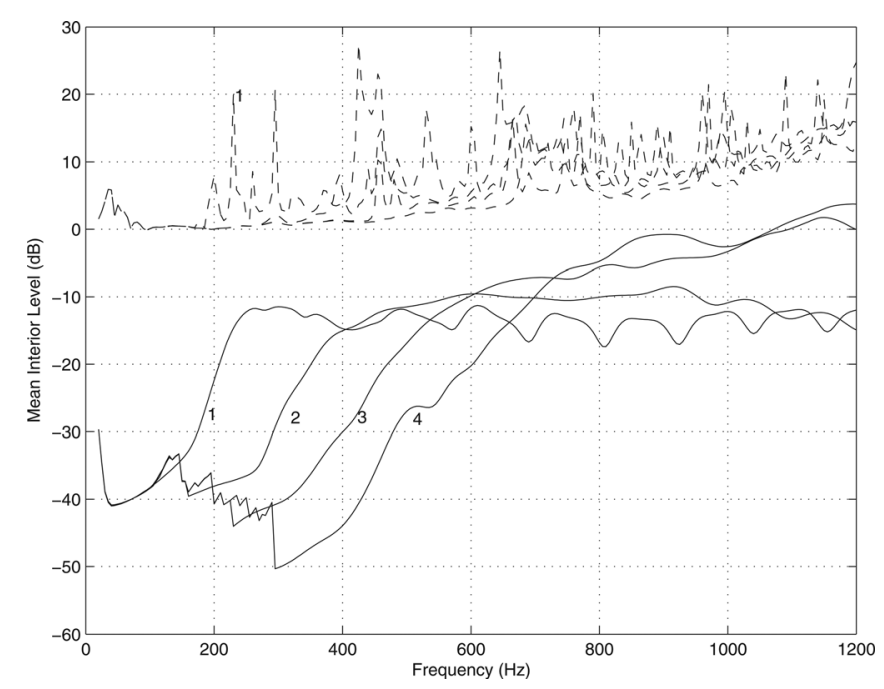

FIG. 12. Exterior reproduction: Interior mean level with interior cancellation (-) and without (-- -). a cylinder, and the behavior is complicated further by the fact that the mode matching order increases with frequency.

\section{CONCLUSIONS}

The performance of sound reproduction systems that make use of higher order loudspeakers has been investigated, for the simple case of $2 \mathrm{D}$ reproduction. It has been verified that a circular array of $N$ th order loudspeakers produces accurate reproduction over $N$ times the radius of a monopole array, or equivalently, over $N$ times the frequency range, with simultaneous cancellation of the exterior sound field. If the requirement for exterior cancellation is removed, the array is able to produce accurate sound fields to $2 N$ times that of the monopole source case. For the complementary case of exterior sound field generation, similar results occur. The increase may be viewed intuitively as occurring because an $N$ th order loudspeaker is able to produce approximately $2 N$ times the spatial variation in the sound field for field radii close to the loudspeaker radius.

The method developed in this paper allows a trade-off between the bandwidth of reproduction and the errors that occur above the spatial Nyquist frequency, by limiting the maximum mode order that is controlled by the array. By reducing the maximum mode order, the spatial Nyquist frequency is reduced slightly, but poor conditioning is avoided and the exterior sound levels that occur above the spatial Nyquist frequency are reduced in amplitude. The limited mode order ensures that the exterior cancellation solutions always produce a lower exterior field than the solutions without exterior control. The exterior level can be further reduced by careful choice of the regularization parameter. A fixed value has been used in the simulations presented here and methods for determining the optimum regularization with frequency have not been considered, but this will be important for producing robust sound reproduction at high frequencies. The alternative solution at high frequencies is to revert to fixed directivity solutions. First order fixed directivity has been considered in Ref. 21, but the capabilities of fixed higher order loudspeakers have yet to be examined.

The average interior reproduction error has been presented for a reproduction radius of $2.5 \mathrm{~m}$, close to the loudspeaker radius, to verify the spatial Nyquist frequency calculations. This produces relatively small bandwidths of accurate reproduction (Fig. 9). In practice, the reproduction radius for a single listener can be less than a tenth of this radius, producing over ten times the bandwidth. For example, an ideal fourth order, exterior cancellation, system consisting of 15 loudspeakers at a radius of $3 \mathrm{~m}$ will provide accurate reproduction over $0.25 \mathrm{~m}$ radius up to $4 \mathrm{kHz}$. At frequencies above the spatial Nyquist frequency of $400 \mathrm{~Hz}$, a reverberant field will be produced within the reproduction region, whose level must be controlled by regularization.

A more general form of higher order sound reproduction system would include the effects of the room surfaces in determining the loudspeaker weights. If a higher order microphone is positioned at the listener position, the spherical harmonic responses of each loudspeaker mode can be measured and the loudspeaker weights determined to produce a desired 
spherical harmonic spectrum. In this case the higher order array uses the room surfaces to improve sound reproduction, for example by reflecting sound off a wall to produce angles of incidence different from the loudspeaker angles. An initial assessment of such systems has been given in Ref. 36. Further work is required to show to what extent the use of room surfaces reduces reproduction error, and how robust reproduction is above the spatial Nyquist frequency of the array.

A relatively small array of higher order loudspeakers is more practical to install in a typical living space than a large array of monopole loudspeakers, suggesting that higherorder arrays can contribute to the improvement of commercial surround systems.

${ }^{1}$ M. Gerzon, "Periphony: With-height sound reproduction," J. Audio Eng. Soc. 21(1), 2-10 (1973).

${ }^{2}$ M. A. Gerzon, "Ambisonics in multichannel broadcasting and video," J. Audio Eng. Soc. 33(11), 859-871 (1985).

${ }^{3}$ J. Daniel, R. Nicol, and S. Moreau, "Further investigations of high order ambisonics and wavefield synthesis for holophonic sound imaging," 114th AES Convention, Amsterdam, Netherlands (March 22-25, 2003), preprint 5788 .

${ }^{4}$ J. Daniel, "Spatial sound encoding including near field effect: Introducing distance coding filters and a viable new ambisonics format," $23 r d$ AES International Conference, Copenhagen, Denmark (May 23-25, 2003).

${ }^{5}$ R. Nicol and M. Emerit, "3D-sound reproduction over an extensive listening area: A hybrid method derived from Holophony and Ambisonic," 16th AES International Conference, Rovaniemi, Finland (April 10-12, 1999).

${ }^{6}$ D. B. Ward and T. D. Abhayapala, "Reproduction of a plane-wave sound field using an array of loudspeakers," IEEE Trans. Speech Audio Process. 9(6), 697-707 (2001).

${ }^{7}$ M. A. Poletti, "Three dimensional surround sound systems based on spherical harmonics," J. Audio Eng. Soc. 53(11), 1004-1025 (2005).

${ }^{8}$ J. Ahrens and S. Spors, "An analytical approach to sound field reproduction using circular and spherical loudspeaker distributions," Acta Acust. Acust. 94, 988-999 (2008).

${ }^{9}$ O. Kirkeby and P. A. Nelson, "Reproduction of plane wave sound fields," J. Acoust. Soc. Am. 94(5), 2992-3000 (1993).

${ }^{10}$ F. M. Fazi, P. A. Nelson, J. E. N. Christensen, and J. Seo, "Surround system based on three dimensional sound field reconstruction," 125th AES Convention, San Francisco, CA (October 2-5, 2008), preprint 7555.

${ }^{11}$ A. J. Berkhout, D. de Vries, and P. Vogel, "Acoustic control by wave field synthesis,” J. Acoust. Soc. Am. 93(5), 2764-2778 (1993).

${ }^{12}$ M. M. Boone, E. N. G. Verheijen, and P. F. Van Tol, "Spatial sound-field reproduction by wave-field synthesis," J. Audio Eng. Soc. 43(12), 1003-1012 (1995).

${ }^{13}$ A. J. Berkhout, D. de Vries, and J. J. Sonke, "Array technology for acoustic wave field synthesis in enclosures," J. Acoust. Soc. Am. 102(5), 2757-2770 (1997).

${ }^{14} \mathrm{D}$. de Vries and M. M. Boone, "Wave field synthesis and analysis using array technology," in Proceedings of the IEEE Workshop on Applications of Signal Processing to Audio and Acoustics, New Paltz, NY (October 1999), pp. 15-18.

${ }^{15}$ S. Takane, Y. Suzuki, and T. Sone, "A new method for global sound field reproduction based on Kirchhoff's integral equation," Acust. Acta Acust. 85, 250-257 (1999).

${ }^{16} \mathrm{~S}$. Ise, "A principle of sound field control based on the KirchhoffHelmholtz integral equation and the theory of inverse systems," Acust. Acta Acust. 85, 78-87 (1999).

${ }^{17}$ M. A. Poletti, F. M. Fazi, and P. A. Nelson, "Sound-field reproduction systems using variable-directivity loudspeakers," J. Acoust. Soc. Am. 129(3), 1429-1438 (2011).

${ }^{18}$ M. A. Poletti and T. D. Abhayapala, "Interior and exterior sound field control using general two dimensional first-order sources," J. Acoust. Soc. Am. 129(1), 234-244 (2011).
${ }^{19}$ M. Unser, "Sampling-50 years after Shannon," Proc. IEEE 88(4), 569-587 (2000).

${ }^{20} \mathrm{H}$. Stark and M. Wengrovitz, "Comments and corrections on the use of polar sampling theorems in CT," IEEE Trans. Acoust., Speech, Signal Process. 31(5), 1329-1331 (1983).

${ }^{21}$ M. A. Poletti, F. M. Fazi, and P. A. Nelson, "Sound-field reproduction systems using fixed-directivity loudspeakers," J. Acoust. Soc. Am. 127(6), 3590-3601 (2010).

${ }^{22} \mathrm{D}$. de Vries, "Sound reinforcement by wavefield synthesis: Adaptation of the synthesis operator to the loudspeaker directivity characteristics," J. Audio Eng. Soc. 44(12), 1120-1131 (1996)

${ }^{23}$ J. Ahrens and S. Spors, "Sound field reproduction employing nonomnidirectional loudspeakers," 126th AES Convention, Munich, Germany (May 7-10, 2009).

${ }^{24}$ M. M. Boone and. O. J. Ouweltjes, "Design of a loudspeaker system with a low-frequency cardiodlike radiation pattern," J. Audio Eng. Soc. 45(9), 702-707 (1997).

${ }^{25} \mathrm{~T}$. Betlehem and T. Abhayapala, "Theory and design of sound field reproduction in reverberant rooms," J. Acoust. Soc. Am. 117, 2100-2111 (2005).

${ }^{26}$ P.-A. Gauthier, A. Berry, and W. Woszczyk, "Sound-field reproduction in-room using optimal control techniques: Simulations in the frequency domain," J. Acoust. Soc. Am. 117(2), 662-678 (2005).

${ }^{27}$ S. Spors, M. Renk, and R. Rabenstein, "Limiting effects of active room compensation using wave field synthesis," 118th AES Convention, Barcelona, Spain (May 28-31, 2005), preprint 6400.

${ }^{28}$ S. Spors, H. Buchner, R. Rabenstein, and W. Herbordt, "Active listening room compensation for massive multichannel sound reproduction systems using wave-domain adaptive filtering," J. Acoust. Soc. Am. 122(1), 354-369 (2007)

${ }^{29}$ L. Fuster, J. J. Lopez, A. Gonzalez, and P. D. Zuccarello, "Room compensation using multichannel inverse filters for wave field synthesis systems," 118th AES Convention, Barcelona, Spain (May 28-31, 2005), preprint 6401.

${ }^{30}$ M. A. Poletti and T. D. Abhayapala, "Spatial sound reproduction systems using higher order loudspeakers," IEEE International Conference on Acoustics, Speech and Signal Processing, ICASSP2011, Prague (May 22-27, 2011).

${ }^{31}$ R. Avizienis, A. Freed, P. Kassakian and D. Wessel, "A Compact 120 Independent Element Spherical Loudspeaker Array with Programmable Radiation Patterns," 120th AES Convention, Paris, France (May 2006), preprint 6783.

${ }^{32}$ B. Rafaely, "Spherical loudspeaker array for local active control of sound," J. Acoust. Soc. Am. 125(5), 3006-3017 (2009).

${ }^{33} \mathrm{~F}$. Zotter and R. Holdrich, "Modeling a spherical loudspeaker system as a multipole source," in Proceedings of the 33rd German Annual Conference on Acoustics, Stuttgart, Germany (March 19-22, 2007).

${ }^{34}$ T. W. Leishman, S. Rollins, and H. M. Smith, "An experimental evaluation of regular polyhedron loudspeakers as omnidirectional sources of sound," J. Acoust. Soc. Am. 120, 1411-1422 (2006).

${ }^{35}$ A. Pasqual, J. R. de F. Arruda, and P. Herzog, "A comparative study of platonic solid loudspeakers as directivity controlled sound sources," in Proceedings of the 2nd International Symposium on Ambisonics and Spherical Acoustics, Paris, France (May 6-7, 2010).

${ }^{36}$ T. Betlehem, C. Anderson and M. A. Poletti, "A directional loudspeaker array for surround sound in reverberant rooms," in Proceedings of the International Conference on Acoustics, Sydney, Australia (August 23-27, 2010).

${ }^{37}$ E. G. Williams, Fourier Acoustics (Academic, San Deigo, 1999), pp. 115-125, 266.

${ }^{38} \mathrm{G}$. N. Watson, A Treatise on the Theory of Bessel Functions, 2nd ed. (Cambridge University Press, Cambridge, 1995), p. 361.

${ }^{39}$ C. P. Mathews and M. D. Zoltowski, "Eigenstructure technique for 2-D angle estimation with uniform circular arrays," IEEE Trans. Signal Process. 42(9), 2395-2407 (1994).

${ }^{40}$ R. Kennedy, P. Sadeghi, T. D. Abhayapala, and H. Jones, "Intrinsic limits of dimensionality and richness in random multipath fields," IEEE Trans. Signal Process. 55(6), 2542-2556 (2007).

${ }^{41}$ A. Edelman, "Eigenvalues and condition numbers of random matrices," SIAM J. Matrix Anal. Appl. 9(4), 543-560 (1988). 